\title{
Power \& Effect: A statistical utility for Macintosh and Windows systems
}

\author{
GLENN E. MEYER \\ Trinity University, San Antonio, Texas
}

\begin{abstract}
Significance tests are not the only step in statistics. Other considerations include effect sizes and adequate sample sizes for a respectable level of statistical power. However, many statistical packages are spotty in their offerings of effect size, complex, and lack a friendly interface. Textbooks may have limited coverage, and calculations entail several formulas and tables. Power \& Effect offers a calculator- and formula-based metaphor to compute popular measures of effect size, simple significance tests between effect sizes, combining of effect sizes, simple significance tests based on known statistical values, and sample size determinations based on predicted results or effect size.
\end{abstract}

Recent reviews suggest that there is more to statistics than tests of significance. Other considerations are measures of effect size or percent of explained variance, the need for adequate sample sizes to guarantee a respectable degree of statistical power, and the need sometimes to compute combined effect sizes or test them for significance (meta-analytic techniques) (Bordens \& Abbot, 1991; Cohen, 1988, 1992; Keppel, 1991; Rosenthal \& Rosnow, 1984). These procedures can be seen as adding insight to a study or as an added burden to the student in introductory statistics.

Although these topics are covered in some introductory statistics and research design texts, there are not always easy ways to carry them out. Texts contain lists of formulas. Sample size calculations entail many steps, the use of complicated charts, and then, more calculations. There can be added nuances for unequal sample sizes or variances, $r$ to $z$ transformations based on natural logarithms, or other arcane procedures. Having taught introductory statistics for many years to audiences with some differences in mathematical skills and in their intrinsic interest in statistics, I do not find it surprising that the average consumer of statistics in psychology may not be too fluent in such techniques. This opinion is echoed in the literature (Cohen, 1977, 1988, 1992; Rosenthal \& Rosnow, 1984).

There would seem to be a need for an easy way to calculate effect sizes and sample sizes to encourage students and researchers to use these techniques. Although some might argue that without hand calculation "one doesn't know where the numbers come from," I disagree. After all, the power charts and calculations are based on already computed tables and sophisticated formulas. A user of such charts does not do the native calculations (Howell,

Trinity University has no commercial interest in this program. For information and reprints, address correspondence to G. E. Meyer, Department of Psychology, Trinity University, 715 Stadium Drive, San Antonio, TX 78212 (e-mail: gmeyer@trinity.edu).

Note: The author has a direct financial interest in the software presented in this paper.-Editor
1992; Keppel, 1991). Similarly, if one uses a program to compute an analysis of variance (ANOVA), one must know that modern programs do not use the computational formulas found in the texts because of needs for numerical accuracy. There are computer packages that offer sophisticated options for power analyses, and some statistics packages have limited options for reporting effect sizes from input data analyses (Meyer, 1993). Although these may be excellent, they are not particularly user friendly, and they are daunting for the novice. With Power \& Effect, I have attempted to design a package that is easy to use. My inspiration came from Cohen (1992), who provided a short and easy method for computing sample sizes.

My objective was to increase the use of analyses of effect size and statistical power. I wanted users to be able to enter a hypothetical study and determine the number of subjects needed for a reasonable level of statistical power, to check easily whether a previously conducted study had an adequate sample size, and to calculate effect sizes from their own work or from the literature.

Using Power \& Effect, for example, students should be able to (1) determine the number of pairs needed for a correlation coefficient to have a statistical power of .80 . Similar calculations can be made for many other procedures and power levels; (2) read an ANOVA table and determine the value of omega ${ }^{2}$ for that study; (3) test several measures of effect size for statistical significance or combine them; and (4) calculate significance for $t$ tests, difference between correlation coefficients, slopes and chi-square if given appropriate statistics.

The program is available for both Macintosh- and Windows-based systems (see Figure 1, A.1-A.3).

\section{DESIGN}

\section{Comprehensive Set of Common Procedures}

A comprehensive set of procedures for the techniques most commonly used in teaching was developed. They are easily accessible through drop-down menus, a hypermedia index, or specific menus for techniques. 

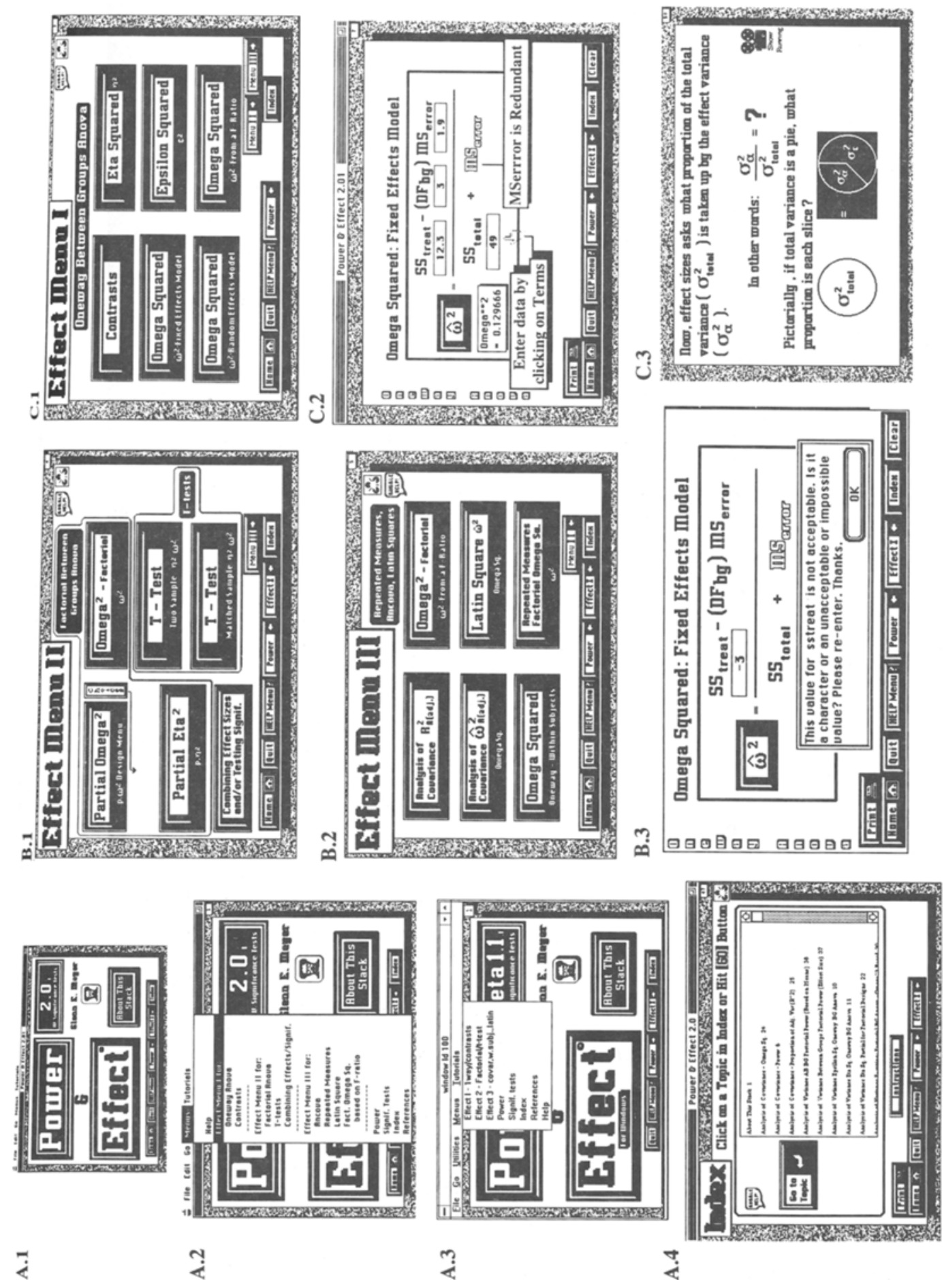

3

茫

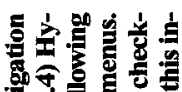

窟

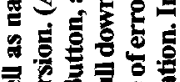

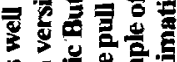

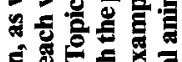

f. 巳

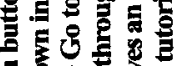

5 홍

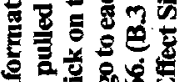

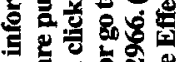

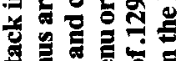

क्⿹

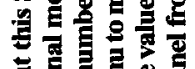

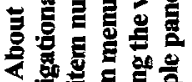

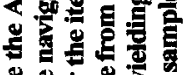

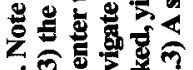

룬

* 녕

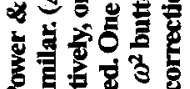

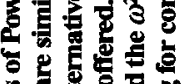

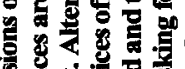

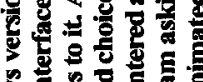

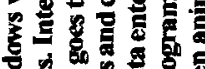

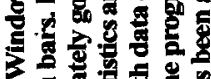

o

西

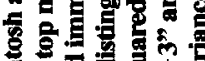

틀

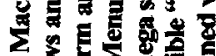
产 象 엉

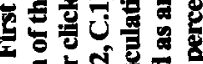

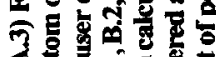

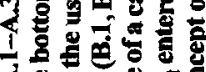

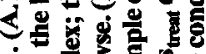
它 
Power \& Effect has two major divisions: Effect Size, which permits calculation of effect sizes from statistics that are input (see Figures 1B-1C), and Sample Size, which allows the calculation of sample sizes from hypothesized outcomes (see Figure 2).

Thus, standard measures of effect size can be calculated if statistics are known. Students or researchers can enter either their own statistics from computer summary tables or those from the works of others. The most common designs are covered. Of particular interest are effect size formulas based on $F$ ratios. These are useful if one wants to evaluate effect size and the work in question only gives an evaluation of significance. The program also contains some simple meta-analytic techniques that allow effect sizes to be combined and/or tested for significance. Effect size techniques are presented in the menus in Figure 1 (B.1-B.3). Sample sizes can be calculated for a set of statistical techniques on the basis of estimates of either the values that one would expect (means, standard deviations, correlations, proportion, etc.) or effect size. (See Figure 2 for the available procedures.)

As an auxiliary function, if one already knows means, standard deviations, frequencies, correlations, or slopes, Power \& Effect offers significance tests for several statistics. These are useful techniques not found in some packages but noted in texts. Also, there are utilities for examining already calculated statistics from other studies. Tests of significance are available for the following:

Pearson's $r$ :

One $r$ versus a Population rho

Simple significance of $r$

One $r$ versus another $r$

$t$ tests

One sample

Two sample

Matched samples

Slope of Regression Lines

Simple significance of a slope

One slope versus a population slope

Slope1 versus Slope2

\section{Effect Size}

These techniques are accessed through the specific menus, index, or drop-down menus. The Power Menu in Figure 2 notes which procedures have the significance test option by marking the buttons.

\section{Graphic User Interface}

A significant problem in. designing software is user friendliness. This is especially the case with statistical software, the novice user of which may be unfamiliar not only with computer usage but with the problem to be solved. Most helpful is the use of a screen metaphor that takes advantage of the user's existing knowledge base and an object-based manipulation strategy (Schneiderman, 1992). Thus, by taking advantage of the user's knowledge base, I have adopted two metaphors to make usage easier. This strategy certainly seems to have been successful in the implementation of the Macintosh Finder and Desktop, Microsoft Windows, and other similar products.

Effect size and the formula metaphor. A hypertext graphic of the formula is used for the technique itself. Thus, in the example given, the user would click on each formula term and a field would appear. After all terms have been entered, the effect size is clicked upon and the result calculated. Extensive error checking, reentry, and correction capabilities are provided. Thus, the user is presented with a form similar to the one presented in a standard text. In Figure 1 (C.2), we see an example in which an omega squared for a fixed effects model is being calculated. The user enters values for $\mathrm{ss}_{\text {treat }}(12.3)$, $\mathrm{DF}_{\mathrm{bg}}(3), \mathrm{ms}_{\text {error }}(1.9)$ and is about to enter $\mathrm{ss}_{\text {total }}$. When these have been entered and the $\omega^{2}$ button is then clicked upon, the value of .12966 will appear. Figure 1 (B.3) shows error correction for an impossible value of a sum of squares term. In this case, "-3" has been entered for $\mathrm{Ss}_{\text {treat }}$, and the program is asking for its correction.

Thus, Power \& Effect offers students, instructors, and other users an easy calculator- and equation-based interface. The user can click on the standard formulas for effect size and enter the appropriate terms. Data entry is congruent with the formulas and examples found in standard texts. The student can see how changing values affect the analyses. The effects of reducing error, adding groups, changing degrees of freedom, and so forth, can be present or tested.

Sample size and the calculator metaphor. We use a calculator metaphor. A panel thus duplicates the statistical information needed for calculating sample size. These are hot hypertext, so that clicking on them opens a field for data entry. Choice of power level and significance level is made with calculator buttons, so that the user is not left pondering what significance level refers to- or what power level to type in. They are prompted. Although to purists this may seem limiting, it aids novices. If a statistical description of a problem is entered in a "virtual" calculator, it will calculate the number of subjects needed. One can easily change parameters and recalculate. The user can see how changing means, standard deviations, sample sizes, power levels, significance levels, and so forth, can affect the analyses and sample sizes.

Figures 2B and 2C show examples of sample size calculations for a two-sample $t$ test and $\mathrm{A} \times \mathrm{B}$ betweengroups ANOVA. In Figure 2B, the user has hypothesized that the means of the two groups would have been approximately 104 and 110, with standard deviations of 12 and 8, respectively. The program takes into account the different standard deviations. An alpha level of .01 was chosen, with a power level of .80 . The program then estimates that 69 subjects are needed per group. In Figure $2 \mathrm{C}$, means for an $\mathrm{A} \times \mathrm{B}$ interaction were entered. The program reports the number of subjects per group for the A main effect, B main effect, and A $\times$ B interaction. These effects are also evaluated as eta squared. 

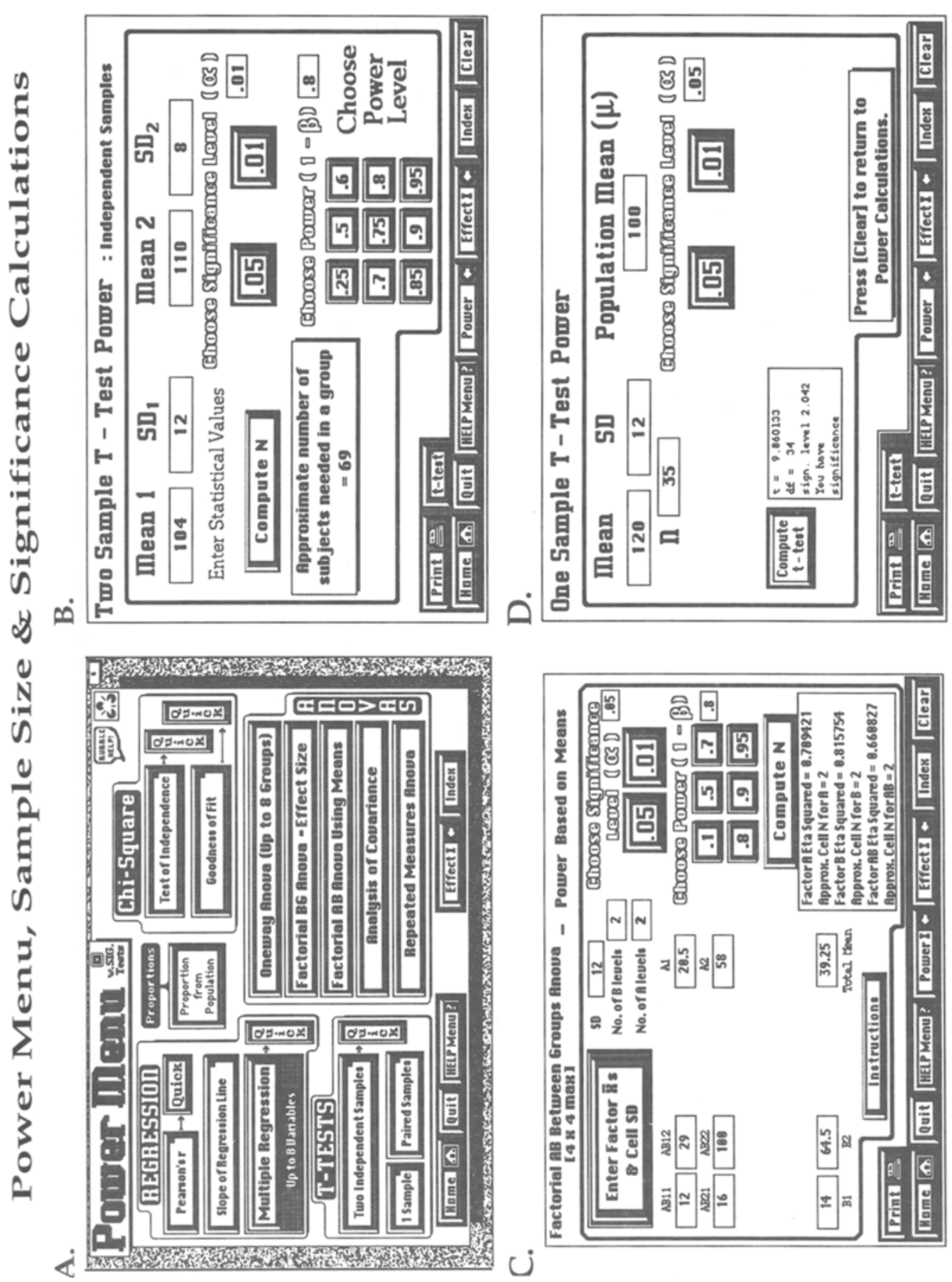

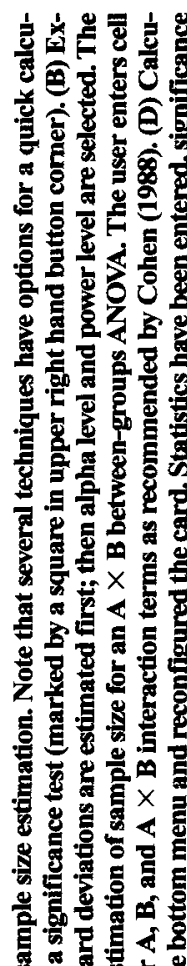

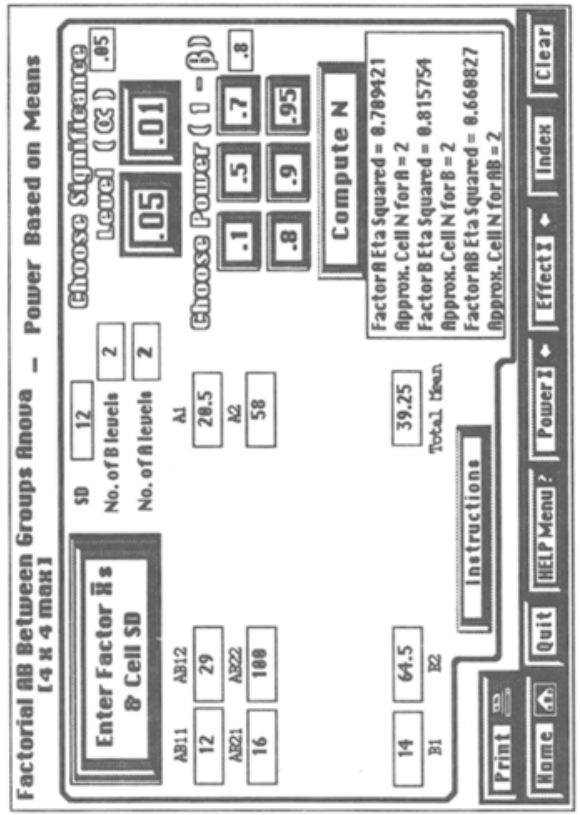

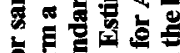

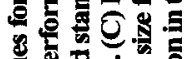

局

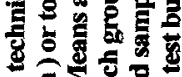

政

空

o.

政

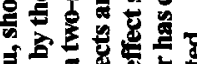

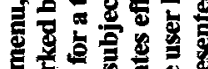

解

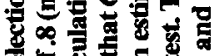

के

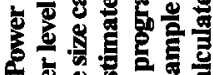

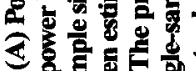

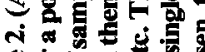

㐘

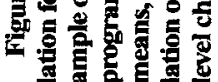


The user can determine the number of subjects that is congruent with which of the three terms is most important for their analyses (Cohen, 1988). Other cards allow exploration of ANOVAs on the basis of effect size estimates rather than mean estimates (see Figure 2A). Figure $2 \mathrm{D}$ demonstrates the program calculating a one-sample $t$ test, given the input of the mean, standard deviation, and sample size. If the user has an already calculated mean, etc., significance can be tested. In class, these are useful for exploring changes in sample statistics and their effect on significance. Also of interest are the comparison procedures for slopes, which are not usually found in packages (see Figure 2A).

These procedures also carry out extensive error testing and give ample opportunities to correct mistaken entries. Examples of errors are given in the Error Menu accessible through the Help Menu.

\section{Navigation, Help, and Tutorials}

Navigation. Power \& Effect contains extensive navigational capacities. It is easy for novices to become trapped in a program and not know where they are, how to move to another place, or how to quit the program. On all windows, a bottom row of buttons enables the user to quit, go to a help menu, to the major technique menus, or to a hypertext subject index that enables one to pick a topic and go to it. These capacities are also duplicated in a set of standard Macintosh pull-down or Windows dropdown menus (see Figure 1 A.1-A.3).

Help and tutorials. The following are available and accessible through the Help Menu:

1. Animated tutorials of general usage, Power windows use, Effect windows use, reviews of effect size and power concepts, and use of the Index. Figure 1 (C.3) presents one panel of the Effect Size animation. The partition of total variance is presented in the graphic. The Power tutorial compares the technical definition of power with a scenario of a physician diagnosing a patient. Also available are animated scenarios of data being entered into effect size or sample size calculations.

2. More complicated procedures have additional instructions available. The instruction button is highlighted when the procedure is opened.

3. Examples of common errors and error messages.

4. Some windows (in the Macintosh version) have a "Bubble Help" facility similar to Balloon Help. When a bubble is activated, cursor movement brings up information about the window. The bubble can be seen in Figure 1 (A.1, B.1, B.2, C.1) and Figure 2A. Unfortunately, the Windows version does not have this facility.

5. A comprehensive, "hot" index of the program's abilities (see Figure 1, A.4).

Thus, one should not need to resort to a manual when the program is in use.

\section{Macintosh and Windows Versions}

Versions of Power \& Effect exist for Macintosh and Windows systems (see Figure 1). The Macintosh version is written in Hypercard 2.1 (it is Hypercard 2.2 compat- ible). A stand-alone version of the program is possible but increases memory size. The Windows version is written in Windowcraft, which is an enhanced version of Hypercard 1.25, but for Windows 3.0 and 3.1. It has a stand-alone version also. Each version has successfully run on machines capable of running System 7 or Windows 3 . Power$\mathrm{PC}$ and Pentium versions seem to work well. Testing has not revealed any significant problems or tendencies to crash. As can be seen in Figure 1, the interfaces are quite similar. Users first seeing the Macintosh version in class but possessing Windows-based machines of their own report little transfer of training problems.

\section{USER TESTING AND EVALUATION}

Both versions of Power \& Effect have been under constant testing and modification for a year. It has been used as a supplement in beginning and advanced undergraduate classes in psychological statistics and in a year-long sequence of advanced statistics for a PsyD program. The undergraduate and graduate classes both used SPSS (UNIX, Macintosh, or PC versions) as their major computational tools, along with statistical calculators. The texts were Howell (1992) and Meyer (1993) for the undergraduates and Keppel (1991) and Meyer (1993) for the graduates. These texts are not lacking in sophistication. Both groups found the interface easy to use and master. Users familiar with Macintosh or Windows were immediately able to navigate and operate. If the users knew a particular statistical technique, they reported that the metaphors were easily comprehended. They were able, when presented analyses or when using their own computer output, to enter data easily and to draw the appropriate conclusions.

\section{Availability}

A more comprehensive description of Power \& Effect is available at no charge by writing or e-mailing the author. Single copies of the program (either version) are available for $\$ 50$. Site licenses are also available.

\section{REFERENCES}

BoRDENS, K. S., \& ABBOTT, B. B. (1991). Research methods and design (2nd ed). Mountain View, CA: Mayfield Press.

CoHEN, J. (1977). Statistical power analysis for the behavioral sciences (rev. ed.). Hillsdale, NJ: Erlbaum.

COHEN, J. (1988). Statistical power analysis for the behavioral sciences (2nd ed.). Hillsdale, NJ: Erlbaum.

COHEN, J. (1992). A power primer. Psychological Bulletin, 112, 155-159.

Howell, D. C. (1992). Statistical methods for psychology (3rd ed.). Boston: PWS-Kent.

KEPPEL, G. (1991). Design and analysis: A researcher's handbook (3rd ed.). Englewood Cliffs, NJ: Prentice-Hall.

MEYER, G. E. (1993). SPSS: A minimalist approach. Fort Worth, TX: Harcourt, Brace, Jovanovich.

RosentHaL, R., \& RosNow, R. L. (1984). Essentials of behavioral research. New York: McGraw-Hill.

SCHNeIderman, B. (1992). Designing the user interface: Strategies for effective human-computer interaction ( 2 nd ed.). Reading, MA: Addison-Wesley.

(Manuscript received November 16, 1994; revision accepted for publication February 6, 1995.) 